\title{
An intrinsic approach to invariants of framed links in 3-manifolds
}

\author{
Efstratia Kalfagianni*
}

\begin{abstract}
We study framed links in irreducible 3-manifolds that are $\mathbb{Z}$-homology 3-spheres or atoroidal $\mathbb{Q}$-homology 3-spheres. We calculate the dual of the Kauffman skein module over the ring of two variable power series with complex coefficients. For links in $S^{3}$ we give a new construction of the classical Kauffman polynomial.
\end{abstract}

Keywords. Characteristic submanifold, framed links, finite type invariants, Kauffman skein module, loop space, Seifert fibered 3-manifolds, toroidal decompositions.

Mathematics Subject Classification (2010). 57N10, 57M2, 57R42, 57R56.

\section{Introduction}

The Kauffman polynomial is a 2-variable Laurent polynomial invariant for links in $S^{3}$ [17] that has interesting applications and connections with contact geometry. The degree in one of the variables of the Kauffman polynomial provides an upper bound for the Thurston-Bennequin norm of Legendrian links [8], [26]. The inequality is known to be sharp for several classes of links (e.g. alternating links) and the proof of this sharpness has led to deeper connections between knot polynomials and contact geometry [25].

In this paper we study framed links in oriented, irreducible 3-manifolds that are $\mathbb{Z}$ homology 3-spheres or atoroidal $\mathbb{Q}$-homology 3-spheres. We give conditions under which an invariant that is defined on framed singular links with one double point gives rise to an invariant of framed links (Theorem 2.6). This allows us to construct formal power series framed link invariants obeying the Kauffman polynomial skein relations. The coefficients of these series are finite type framed link invariants and are perturbative versions of the Reshetikhin-Turaev, Witten SO(n)-invariants [24], [29] in the sense of Le-Murakami-Ohtsuki [20]. Using weight systems corresponding to appropriate representations of the Lie algebras $\operatorname{so}(n)$ and the naturality of the LMO invariant, one obtains a Kauffman type power series invariant for framed links in all

\footnotetext{
*Supported in part by NSF grant DMS-0805942.
} 
Q-homology 3-spheres. Our approach in this paper is quite different from this line and allows us to solve the subtler problem of constructing power series invariants with given values on a set of initial links. Our approach here, that exhibits the interplay between skein framed link theory and the topology of 3-manifolds, is inspired by the study of Vassiliev invariants (also known as finite type invariants) [27] using 3-dimensional topology techniques [14]. The precise relation of the power series constructed here to the one obtained via the LMO invariant is not clear to us at this point.

Definition 1.1. Let $M$ be an oriented $\mathbb{Q}$-homology 3-sphere. A framed $m$-component link is a collection of $m$ unordered (unoriented) circles smoothly and disjointly embedded in $M$ and such that each component is equipped with a continuous unit normal vector field. Two framed links are equivalent if they are isotopic by an ambient isotopy that preserves the homotopy class of the vector field on each component. Let $\overline{\mathscr{L}}:=\overline{\mathscr{L}}(M)$ denote the set of isotopy classes of framed links in $M$.
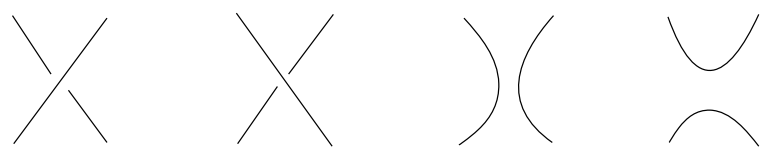

Figure 1. The parts of $L_{+}, L_{-}$and $L_{o}$ and $L_{\infty}$ in $B$.

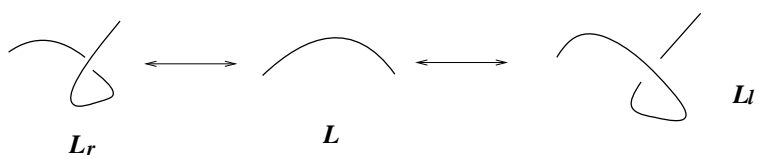

Figure 2. $L_{r}$ and $L_{l}$ are obtained by a full twist from $L$.

To state the main result of the paper we need some notation and conventions: Let $L_{+}, L_{-}, L_{o}$ and $L_{\infty}$ denote four framed links that are identical everywhere except in a 3-ball $B$ in $M$. There under a suitable projection of the parts in $B, L_{+}, L_{-}, L_{o}$ and $L_{\infty}$ look as shown in Figure 1. Also for every framed link we denote by $L_{r}, L_{l}$ the framed links that are identical to $L$ everywhere except in a 3-ball where they differ as shown in Figure 2. Here we suppose that the orientation of $M$ agrees with the right-handed orientation of the 3-balls containing the link parts in Figures 1 and 2 and that the framing vector for link parts in these figures is perpendicular to the page. The framings of the links coincide everywhere outside the parts shown in Figures 1 and 2.

Let $\hat{\Lambda}:=\mathbb{C}[[x, y]]$ denote the ring of formal power series in $x, y$ over $\mathbb{C}$ and let $t:=e^{x}=1+x+\frac{x^{2}}{2}+\cdots$. Let us set $a:=i e^{y}=i+i y+\frac{i y^{2}}{2}+\cdots$ and set $z:=i t-(i t)^{-1}=i e^{x}+i e^{-x}=2 i+i x^{2}+\cdots$. Note that $a$ and $z$ are invertible in $\hat{\Lambda}$. 
Definition 1.2. The Kauffman skein module of $M$ over $\hat{\Lambda}$, denoted by $\mathfrak{F}(M)$, is the quotient of the free $\hat{\Lambda}$-module with basis $\overline{\mathscr{L}}$ by its ideal generated by all the relations of the following two types:

$$
\begin{gathered}
L_{+}-L_{-}=z\left[L_{o}-L_{\infty}\right], \\
L_{r}=a L \quad \text { and } \quad L_{l}=a^{-1} L .
\end{gathered}
$$

We will use $\mathfrak{F}^{*}(M):=\operatorname{Hom}_{\hat{\Lambda}}(\mathfrak{F}(M), \hat{\Lambda})$ to denote the $\hat{\Lambda}$-dual of $\mathfrak{F}(M)$.

Remark 1.3. The usual convention in skein module theory is to allow an empty link as part of the set $\overline{\mathscr{L}}$. In contrast to that, in this paper, we find it convenient to work with non-empty links (Definition 1.1).

Remark 1.4. Since the links are unoriented the declarations $L_{+}$and $L_{-}$, when considering a crossing, are arbitrary. However this does not matter for our purposes since the first skein relation in Definition 1.2 is invariant under simultaneously interchanging $L_{+}$with $L_{-}$and $L_{o}$ with $L_{\infty}$.

To continue let $\pi:=\pi(M)$ denote the set of non-trivial conjugacy classes of $\pi_{1}(M)$ and $\hat{\pi}$ denote the set obtained from $\pi$ by identifying the conjugacy class of every element $1 \neq x \in \pi_{1}(M)$ with that of $x^{-1}$. Also let $S(\hat{\pi})$ denote the symmetric algebra of the free $\hat{\Lambda}$-module, say $\hat{\Lambda} \hat{\pi}$, with basis $\hat{\pi}$. Finally, let $S^{*}(\hat{\pi}):=$ $\operatorname{Hom}_{\hat{\Lambda}}(S(\hat{\pi}), \hat{\Lambda})$ denote the $\hat{\Lambda}$-dual of $S(\hat{\pi})$.

Theorem 1.5. Let $M$ be an oriented $\mathbb{Q}$-homology 3-sphere with $\pi_{2}(M)=0$ and such that if $H_{1}(M) \neq 0$ then $M$ is atoroidal. Then there is a $\hat{\Lambda}$-module isomorphism

$$
\mathfrak{F}^{*}(M) \cong S^{*}(\hat{\pi}) .
$$

For components that are homologically trivial in $M$ the homotopy class of the framing vector field is determined by an integer: the algebraic intersection number of a push-out of the component in the direction of the framing vector field with a Seifert surface bounded by the component. This algebraic intersection number is the self-linking number of the component. There is a canonical framing defined by the Seifert surface that corresponds to the integer zero. This implies that in a $\mathbb{Z}$ homology sphere, for every underlying (unframed) isotopy class of knots the framed knot types correspond to integers. The self-linking number can also be defined in terms of Vassiliev-Gusarov axioms; it is a finite type framed link invariant of order one. As shown by Chernov [3] this point of view generalizes to all framed knots in 3-manifolds; in particular for knots in irreducible $\mathbb{Q}$-homology 3-spheres that we study here. For $M$ as above, given a conjugacy class $c$ in $\pi_{1}(M)$ and a fixed framed knot $C K$ representing $c$, Chernov shows that there is a unique $\mathbb{Z}$-valued invariant for all framed knots representing $c$ with given value on $C K$ (Theorem 2.2 of [3]). His work implies that, with a chosen set of initial knots, for every underlying (unframed) 
isotopy class of knots the framed knot types correspond to integers. This point will be useful to us in the next sections.

The isomorphism in Theorem 1.5 also depends on a choice of initial links which we now discuss: For every unordered sequence of elements in $\hat{\pi} \cup\{1\}$ we choose a framed link $C L$ that realizes it and call it an initial link. For elements in $\hat{\pi} \cup\{1\}$ that are trivial in $H_{1}(M)$ we choose the canonical framing. This means that the integer describing the framing on each component of an initial link is zero. For an initial link $C L$ with $k$ homotopically trivial components we choose $C L=C L^{*} \sqcup U^{k}$, where $C L^{*}$ is an initial link with no homotopically trivial components and $U^{k}$ is the standard unlink in a 3-ball disjoint from $C L^{*}$. The one component unlink $U^{1}$ will be abbreviated to $U$. In general we will assume that each component of an initial link $C L$ is the chosen initial knot for the corresponding element in $\hat{\pi} \cup\{1\}$. We will also assume that each component is the initial knot required to define Chernov's self-linking invariant. We will denote by $\mathscr{C L}^{*}$ the set of all initial links with no homotopically trivial components.

The elements in the set $\mathscr{C L}^{*} \cup\{U\}$ are in one-to-one correspondence with a basis of $S(\hat{\pi})$. An element $R_{M} \in \mathfrak{F}^{*}(M)$ gives rise to one in $S^{*}(\hat{\pi})$ by restriction on the set $\mathscr{C L}^{*} \cup\{U\}$. Theorem 1.5 will follow easily once we have proven the following result (see Section 4 for details).

Theorem 1.6. Let $M$ be an oriented $\mathbb{Q}$-homology 3-sphere with $\pi_{2}(M)=0$, and such that if $H_{1}(M) \neq 0$ then $M$ is atoroidal. Given a map $\mathcal{R}_{M}: \mathscr{C L}^{*} \cup\{U\} \rightarrow \hat{\Lambda}$ there exists a unique map $R_{M}: \overline{\mathscr{L}} \rightarrow \hat{\Lambda}$ such that:

(1) The restriction of $R_{M}$ on $\mathcal{C L}^{*} \cup\{U\}$ is equal to $\mathcal{R}_{M}$.

(2) $R_{M}$ satisfies the Kauffman skein relation

$$
R_{M}\left(L_{+}\right)-R_{M}\left(L_{-}\right)=z\left[R_{M}\left(L_{o}\right)-R_{M}\left(L_{\infty}\right)\right]
$$

for every skein quadruple of links $L_{+}, L_{-}, L_{o}$ and $L_{\infty}$ as in Figure 1.

(3) $R_{M}\left(L_{r}\right)=a R_{M}(L)$ and $R_{M}\left(L_{l}\right)=a^{-1} R_{M}(L)$ for every $L \in \overline{\mathscr{L}}$.

Let $\Lambda:=\mathbb{C}\left[a^{ \pm 1}, z^{ \pm 1}\right]$ denote the ring of Laurent polynomials in $a$ and $z$. We can define the Kauffman skein module of $M$ over $\Lambda$, denoted by $\mathfrak{F}_{\Lambda}(M)$, and consider its $\Lambda$-dual, $\mathfrak{F}_{\Lambda}^{*}(M):=\operatorname{Hom}_{\Lambda}\left(\mathfrak{F}_{\Lambda}(M), \Lambda\right)$. As we will discuss in Section 4 , for links in $S^{3}$, if we choose the value $R_{S^{3}}(U)$ to lie in $\Lambda$ then $R_{S^{3}}(L) \in \Lambda$, for every $L \in \overline{\mathscr{L}}$. This implies that $\mathfrak{F}_{\Lambda}^{*}\left(S^{3}\right) \cong \Lambda$ and leads to the following question:

Question. Let $M$ be as in Theorem 1.5. Can we choose the initial links $C L^{*} \in \mathcal{C} \mathscr{L}^{*}$ so that we have a $\Lambda$-module isomorphism

$$
\mathfrak{F}_{\Lambda}^{*}(M) \cong S_{\Lambda}^{*}(\hat{\pi}) ?
$$

Here, $S_{\Lambda}^{*}(\hat{\pi})$ denotes the $\Lambda$-dual of the symmetric algebra of the free $\Lambda$-module with basis $\hat{\pi}$. 
In [16] we constructed formal power series invariants that satisfy the HOMFLY skein change formula for unframed oriented links in large classes of $\mathbb{Q}$-homology 3-spheres. Cornwell [4], [5], [6] shows that for lens spaces both the question above and its analogue for the HOMFLY skein module of [15] have a positive answer. As a result he obtains analogues of the aforementioned results of [8], [26] for Legendrian links in contact lens spaces.

Theorem 2.6 of this paper is the framed link analogue of the "integrability of singular link invariants" results proved in [14], [16]. Theorem 2.6 does not follow from the results in these papers: In [14] we only treat knots while in [16] we treat links in some classes of irreducible $\mathbb{Z}$-homology 3-spheres. In this paper we are able to remove those restrictions and deal with all irreducible $\mathbb{Z}$-homology 3 -spheres; see Theorem 3.1 and Remark 3.2. If one forgets the framing, Theorem 3.1 generalizes the integrability results and Theorem A of [16] for links in all irreducible $\mathbb{Z}$-homology 3-spheres.

Framed links in general 3-manifolds and their skein modules were studied by several authors before; see [23] and references therein. In particular, Przytycki [22] introduced a two term homotopy skein module of framed links in oriented 3-manifolds as quantum deformation of the fundamental group. In [13] Kaiser calculated this module over the ring of Laurent polynomials with $\mathbb{Z}$-coefficients. He showed that if a 3-manifold contains no non-separating 2-spheres or tori then Przytycki's module is a symmetric algebra of the free module with basis the set of non-trivial conjugacy classes of $\pi_{1}(M)$. Kaiser also studied several variations of two term skein modules and put the classical self-linking number for null homologous knots as well as Chernov's generalization of it in the skein module theory framework. For details the reader is referred to [13].

The paper is organized as follows: In Section 2 we formulate the problem of integrating framed singular link invariants to invariants of framed links. Then we state an integrability theorem and prove it for atoroidal $\mathbb{Q}$-homology spheres. In Section 3 we treat manifolds containing essential tori and in Section 4 we construct the Kauffman power series invariants and prove Theorems 1.5 and 1.6.

Throughout the paper we will work in the smooth category.

Acknowledgment. I thank Chris Cornwell for his interest in this work and for several stimulating questions about link theory in 3-manifolds that motivated me to go back and work on this project. I thank Vladimir Turaev for suggesting that I formulate the main result of the paper in terms of skein modules. I am grateful to the anonymous referees for reading the paper carefully and making thoughtful comments and suggestions that helped me improve the exposition. 


\section{Framed oriented singular link invariants}

Throughout this section we will work with oriented links in oriented 3-manifolds. Theorem 2.6, as well as its unframed counterparts [14], [16], [21], are proved for oriented links in oriented 3-manifolds. For example, the definitions of the signs of resolutions of double points below use the orientation of links as well as that of the ambient 3-manifolds.

2.1. Framed oriented singular links and resolutions. Let $M$ be an oriented $\mathbb{Q}$ homology 3-sphere. An $m$-component oriented framed singular link of order $n$ is a collection of unordered oriented circles, smoothly immersed in $M$ such that (i) the only singularities are exactly $n$ transverse double points; and (ii) the image of each component is equipped with a continuous unit normal vector field. We consider framed singular links up to ambient isotopy that preserves the orientations, the transversality of the double points and the homotopy class of the vector field on each component. For $n=0$ we have an oriented framed link. We will denote by $\mathscr{L}^{(n)}:=\mathscr{L}^{(n)}(M)($ resp. $\mathscr{L}:=\mathscr{L}(M))$ the set of isotopy classes of oriented framed singular links of order $n$ (resp. links) in $M$.

Convention. To simplify the exposition, for the remaining of the section and the next section, we will say a framed link (resp. singular link) to mean an oriented framed link (resp. singular link). Also when we say a 3-manifold, we will mean an oriented 3-manifold.

Let $P$ denote a disjoint union of oriented circles and consider a framed singular link represented by a smooth immersion $L: P \rightarrow M$. Let $p \in M$ be a double point of $L$; the inverse image consists of two points $p_{1}, p_{2} \in P$. There are disjoint intervals $\sigma_{1}$ and $\sigma_{2}$ on $P$ with $p_{i} \in \operatorname{int}\left(\sigma_{i}\right), i=1,2$, such that for a neighborhood $B$ of $p$ we have $L \cap B=L\left(\sigma_{1}\right) \cup L\left(\sigma_{2}\right)$. Moreover, there is a proper 2-disc $D$ in $B$ such that $L\left(\sigma_{1}\right), L\left(\sigma_{2}\right) \subset D$ intersect transversally at $p$. Now $L\left(\sigma_{1}\right) \cup L\left(\sigma_{2}\right)$ intersects $\partial D$ at four points and, since $\sigma_{i}$ inherits an orientation from that of $P$, we can talk of the initial and terminal point of $L\left(\sigma_{i}\right)$. Choose $\operatorname{arcs} a_{1}, a_{2}, b_{1}, b_{2}$ with disjoint interiors such that

(1) $a_{1}$ and $a_{2}$ go from the initial point of $L\left(\sigma_{1}\right)$ to the terminal point of $L\left(\sigma_{1}\right)$ and lie in distinct components of $\partial B \backslash \partial D$; and

(2) $b_{1}$ and $b_{2}$ lie on $\partial D$ with $b_{1}$ going from the initial point of $L\left(\sigma_{1}\right)$ to the terminal point of $L\left(\sigma_{2}\right)$ and $b_{2}$ from the initial point of $L\left(\sigma_{2}\right)$ to the terminal point of $L\left(\sigma_{1}\right)$. The complement of $b_{1} \sqcup b_{2}$ in $\partial D$ consists of two arcs, say $c_{1}, c_{2}$.

The orientation of $M$ and that of $L\left(\sigma_{2}\right)$ define an orientation of $a_{1} \sqcup a_{2}$; suppose that this induced orientation agrees with the one of $a_{1}$ and is opposite to that of $a_{2}$. Define the positive resolution of $L$ at $p$ to be

$$
L_{+}=\overline{L \backslash L\left(\sigma_{2}\right)} \cup a_{1},
$$


and the negative resolution to be

$$
L_{-}=\overline{L \backslash L\left(\sigma_{2}\right)} \cup a_{2} .
$$

In the case that $n=1$ we also define

$$
\begin{aligned}
L_{o} & =\overline{L \backslash\left(L\left(\sigma_{2}\right) \cup L\left(\sigma_{1}\right)\right)} \cup\left(b_{1} \sqcup b_{2}\right), \\
L_{\infty} & =\overline{L \backslash\left(L\left(\sigma_{2}\right) \cup L\left(\sigma_{1}\right)\right)} \cup\left(c_{1} \sqcup c_{2}\right) .
\end{aligned}
$$

Note that $L_{\infty}$ only makes sense as an unoriented link.

Definition 2.1. A framed singular link $L$ is called inadmissible if there is a 2-disc $D \subset$ $M$ such that $L \cap D=\partial D$ and exactly one double point of $L$ lies on $\partial D$. Otherwise the singular link is called admissible. A crossing change on a link that produces an inadmissible singular link as intermediate step will be called an inadmissible crossing change.

In the proof of Theorem 2.6 it will be convenient for us to work with framed links with ordered components: Let $\widetilde{\mathscr{L}}$ denote the set of isotopy classes of such framed links in $M$. Similarly, let $\widetilde{\mathscr{L}}^{(n)}$ denote the set of isotopy classes of ordered framed singular links with $n$-double points. There is an obvious map $r: \tilde{\mathscr{L}} \rightarrow \mathscr{L}$ that forgets the ordering of the components of links; similarly we have forgetful maps $\mathrm{r}_{n}: \tilde{\mathscr{L}}^{(n)} \rightarrow \mathscr{L}^{(n)}$ for all $n \in \mathbb{N}$. Recall from the Introduction that the framing of a knot is determined by an integer, where in the case of not homologically trivial knots this integer is provided by Chernov's work. Thus the framing of an $m$-component link in $\widetilde{\mathscr{L}}$ is determined by an ordered sequence $\left\{\mathbf{f}_{1}, \ldots, \mathbf{f}_{m}\right\}$ of $m$ integers; one assigned to each component of the link. Every entry of the sequence is the affine self-linking number of a link component and it changes by 2 under an inadmissible crossing change while it remains unchanged under admissible crossing changes (Theorems 2.2, [3]). Then, via $\mathfrak{r}$, an unordered link $L \in \mathscr{L}$ inherits an unordered sequence of integers: More specifically, given $L \in \mathscr{L}$, there is a set of ordered integer sequences, say $\mathbf{f}$, corresponding to elements in $r^{-1}(L)$. We assign to $L$ the map

$$
\mathrm{r}^{-1}(L) \rightarrow \mathbf{f},
$$

sending each element to its corresponding ordered sequence. We will often abuse the terminology and refer to $\mathbf{f}$ as the framing of the link $L$.

Definition 2.2. The total framing of a link $L \in \mathscr{L}$ is defined to be $\tau(L):=\sum_{i=1}^{m} \mathbf{f}_{\mathbf{i}}$ where $\left\{\mathbf{f}_{1}, \ldots, \mathbf{f}_{m}\right\}$ is the ordered sequence corresponding to an appropriate lift $\widetilde{L} \in \mathrm{r}^{-1}(L)$ of $L$.

Definition 2.3. For an ordered, framed singular link $\widetilde{L}_{\times} \in \widetilde{\mathscr{L}}^{(1)}$ we define a sequence of integers $\left\{\mathbf{f}_{1}, \ldots, \mathbf{f}_{m}\right\}$ by

$$
\mathbf{f}_{i}\left(\tilde{L}_{\times}\right):= \begin{cases}\mathbf{f}_{i}\left(\tilde{L}_{+}\right)-\mathbf{f}_{i}\left(\tilde{L}_{-}\right) & \text {if } \times \in \tilde{L}_{i} \\ \mathbf{f}_{i}\left(\tilde{L}_{+}\right)=\mathbf{f}_{i}\left(\tilde{L}_{-}\right) & \text {otherwise }\end{cases}
$$


Note that, in the first case, $\mathbf{f}_{i}\left(\tilde{L}_{\times}\right)$is non-zero only if $L_{\times}$is inadmissible, in which case it is equal to 2 . For an unordered singular link $L_{\times} \in \mathscr{L}^{(1)}$ we have a set of ordered integer sequences, say $\mathbf{f}$, corresponding to elements in $\mathrm{r}^{-1}\left(L_{\times}\right)$. The map $\mathrm{r}^{-1}\left(L_{\times}\right) \rightarrow \mathbf{f}$, assigning to every ordered link in that preimage its corresponding sequence, gives an unordered sequence of integers for $L_{\times}$.

2.2. Integration of singular link invariants. Given an abelian group $\mathbb{A}$ and a framed link invariant $F: \mathscr{L} \rightarrow \mathbb{A}$, we can extend it to an invariant of framed singular links by defining

$$
f\left(L_{\times}\right):=F\left(L_{+}\right)-F\left(L_{-}\right),
$$

for every $L_{\times} \in \mathscr{L}^{(1)}$. Continuing inductively we can extend the invariant on singular links in $\mathscr{L}^{(n)}$ for all $n \in \mathbb{N}$. We are interested in reversing this process; the reverse process is usually referred to as integration of the singular link invariant to an invariant of links [1], [14], [16], [21]. In this section we deal with the following question: Suppose that we are given an invariant of framed singular links $f: \mathscr{L}^{(1)} \rightarrow \mathbb{A}$. Under what conditions is there a framed link invariant $F: \mathscr{L} \rightarrow \mathbb{A}$ so that (1) holds for all singular links $L_{\times} \in \mathscr{L}^{(1)}$ ? We will address this question for links in $\mathbb{Q}$-homology 3-spheres with trivial $\pi_{2}$.

Definition 2.4. Let $N$ be an oriented compact 3-manifold with or without boundary. A map $\Phi: S^{1} \times S^{1} \rightarrow N$ is called essential if it induces an injection on $\pi_{1}$ and it cannot be homotoped to a map $\Phi^{\prime}: S^{1} \times S^{1} \rightarrow \partial N$. Otherwise $\Phi$ is called inessential. The manifold $N$ is called atoroidal if there are no essential maps $S^{1} \times S^{1} \rightarrow N$.

Remark 2.5. Let $L_{\times \times} \in \mathscr{L}^{(2)}$ be a framed singular link with two inadmissible singular points. By resolving the singular points, one at a time, we obtain four singular links in $\mathscr{L}^{(1)}$. These are shown in Figure 3, where the notation is consistent with that of Figure 2. We note that $L_{\times r}$ is equivalent to $L_{r \times}$. Similarly, $L_{\times l}$ is
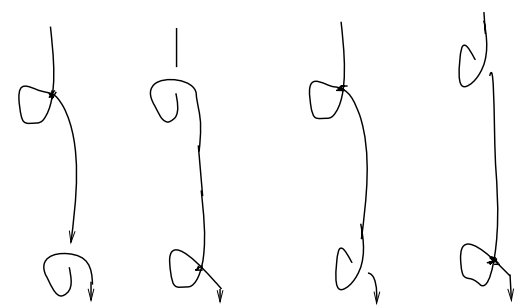

Figure 3. From left to right: $L_{\times r}, L_{r \times}, L_{\times l}, L_{l \times}$.

equivalent to $L_{l \times}$. Thus if $f: \mathscr{L}^{(1)} \rightarrow \mathbb{A}$ is an invariant of framed singular links then we have

$$
f\left(L_{\times r}\right)=f\left(L_{r \times}\right) \quad \text { and } \quad f\left(L_{\times l}\right)=f\left(L_{l \times}\right) .
$$


Now (2) implies that the signed sum of $f$ on the four singular links in Figure 3, where signs are determined by (1), is equal to zero. Next we will show that if this holds true for all $L_{x \times} \in \mathscr{L}^{(2)}$, then $f$ can be integrated to a framed link invariant.

Theorem 2.6. Suppose that $M$ is a $\mathbb{Q}$-homology sphere with $\pi_{2}(M)=0$ and such that if $H_{1}(M) \neq 0$ then $M$ is atoroidal. Let $f: \mathscr{L}^{(1)} \rightarrow \mathbb{A}$ be an invariant of framed singular links with one double point. Suppose that $\mathbb{A}$ is torsion free and that the invariant $f$ satisfies the relation

$$
f\left(L_{\times+}\right)-f\left(L_{\times-}\right)=f\left(L_{+\times}\right)-f\left(L_{-\times}\right)
$$

for every $L_{x \times} \in \mathscr{L}^{(2)}$. Then there exists a framed link invariant $F$ such that $f$ is derived from $F$ via equation (1). Here, the four singular links appearing in (3) are obtained by resolving the singular points of $L_{\times \times}$one at a time.

Theorem 2.6 is the framed link analogue of Theorem 3.16 of [14] and Theorem 3.1.2 of [16]. As explained in the Introduction, however, here we work in a more general class of manifolds. Also the presence of framing requires an adaptation of the arguments: to formulate the correct "global integrability condition" (equation (6) below) we need a notion of global framing around homotopies of links. The definition of such a notion is facilitated by the works of Chernov and Kaiser [3], [13] (Definition 2.7). For arguments that are very similar to these in [14], [16] we will refer the reader to these articles for details.

2.3. Loop space and framing control. Because in this section we work with oriented links we need to slightly modify the set of initial links $\mathcal{C L}^{*} \cup\{U\}$ chosen in the Introduction. Recall that $\mathscr{L}$ (resp. $\overline{\mathscr{L}}$ ) denotes the set of isotopy classes of framed oriented (resp. unoriented) links in $M$. Consider the set of oriented links $\mathcal{C} \mathscr{L}:=\mathfrak{v}^{-1}\left(\mathcal{C L}^{*} \cup\{U\}\right)$, where $\mathfrak{v}: \mathscr{L} \rightarrow \overline{\mathscr{L}}$ is the obvious forgetful map. Also recall that $\tilde{\mathscr{L}}$ denotes the set of isotopy classes of ordered framed links in $M$ and that we defined a forgetful map $\mathrm{r}: \widetilde{\mathscr{L}} \rightarrow \mathscr{L}$. Given $C L \in \mathscr{C} \mathscr{L}$, we pick $L \in \mathrm{r}^{-1}(\mathcal{C} \mathscr{L})$. We will also use $L$ to denote a representative $L: P \rightarrow M$ of $L$, where $P$ is a disjoint union of oriented circles. Let $\mathcal{M}^{L}(P, M)$ denote the space of ordered smooth framed immersions $P \rightarrow M$ homotopic to $L$, equipped with the compact-open topology. For every $L^{\prime} \in \tilde{\mathscr{L}}$ and representative $L^{\prime} \in \mathcal{M}^{L}(P, M)$, let $\Phi: P \times[0,1] \rightarrow M$ be a homotopy with $\Phi(P \times\{0\})=L^{\prime}$ and $\Phi(P \times\{1\})=L$. After a small perturbation we can assume that for only finitely many points $0<t_{1}<t_{2}<\cdots<t_{n}<1$, $\phi_{t}:=\Phi(P \times\{t\})$ is not an embedding and it is a singular framed link of order 1. For different $t^{\prime} s$ in an interval of $[0,1] \backslash\left\{t_{1}, t_{2}, \ldots, t_{n}\right\}$ the corresponding framed links are equivalent and when $t$ passes through $t_{i}, \phi_{t}$ changes from one resolution of $\phi_{t_{i}}$ to the other.

For $C L \in \mathcal{C L}$, let $\mathcal{M}^{C L}(M)$ denote the space of unordered smooth framed immersions homotopic to $C L$, equipped with the compact-open topology. The projection 
$\mathfrak{q}: \mathcal{M}^{L}(P, M) \rightarrow \mathcal{M}^{C L}(M)$ is a covering map away from points that are fixed under permutation of components.

Definition 2.7. Let $\Phi$ be a homotopy between ordered links $L_{1}, L_{2} \in \mathcal{M}^{L}(P, M)$ with points $0<t_{1}<\cdots<t_{n}<1$ such that $\phi_{t_{j}} \in \widetilde{\mathscr{L}}^{(1)}$. For each singular link $\phi_{t_{j}}$ we have a sequence $\left\{\mathbf{f}_{i}^{j} \mid i=1, \ldots, m\right\}$ as in Definition 2.3. We define the total framing of $\Phi$ to be the sequence of integers $\left\{\Delta \mathbf{f}_{i} \mid i=1, \ldots, m\right\}$, where

$$
\Delta \mathbf{f}_{i}:=\sum_{j=1}^{n} \delta_{j}^{i} \varepsilon_{j} \mathbf{f}_{i}^{j}\left(\phi_{t_{j}}\right)
$$

Here $\delta_{j}^{i}=1$ if the $i$-th component of $\phi_{t_{j}}$ contains the double point and 0 otherwise. Also $\varepsilon_{j}=1$ if $\phi_{t_{j}+\delta}$, for $\delta>0$ sufficiently small, is a positive resolution of $\phi_{t_{j}}$ and $\varepsilon_{j}=-1$ otherwise. We will say that the total framing is zero iff $\Delta \mathbf{f}_{i}=0$, for all $1, \ldots, m$.

Given a loop $\Phi \in \mathcal{M}^{C L}(M)$ we obtain a set of ordered sequences $\Delta \mathbf{f}_{\Phi}$ associated to the set of all lifts of $\Phi$ in $\mathcal{M}^{L}(P, M)$. The map $\mathfrak{q}^{-1}(\Phi) \rightarrow \Delta \mathbf{f}_{\Phi}$ defines an unordered sequence of integers for $\Phi$. The homotopy $\Phi$ is called framing preserving iff the total framing of every element in $\mathfrak{q}^{-1}(\Phi)$ is zero. We will write $\Delta \mathbf{f}_{\Phi}=\mathbf{0}$.

2.4. Beginning the proof of Theorem 2.6. We want to define an invariant $F: \mathscr{L} \rightarrow$ $\mathbb{A}$ that is obtained from the given $f: \mathscr{L}^{(1)} \rightarrow \mathbb{A}$ via (1). First we assign values of $F$ on the set of initial links $\mathscr{C} \mathscr{L}$. Now fix $C L \in \mathcal{C L}$ and let $L^{\prime} \in \mathcal{M}^{C L}(M)$ be a framed link. Choose a generic homotopy $\Phi$ from $L^{\prime}$ to $C L$. Let $0<t_{1}<t_{2}<\cdots<t_{n}<1$ denote the points where $\phi_{t}$ is not an embedding. Recall that $\phi_{t_{i}} \in \mathscr{L}^{(1)}$ such that for different $t^{\prime} s$ in an interval of $[0,1] \backslash\left\{t_{1}, t_{2}, \ldots, t_{n}\right\}$, the corresponding framed links are equivalent. When $t$ passes through $t_{i}, \phi_{t}$ changes from one resolution of $\phi_{t_{i}}$ to another. We define

$$
F\left(L^{\prime}\right)=F(C L)+\sum_{i=1}^{n} \varepsilon_{i} f\left(\phi_{t_{i}}\right)
$$

Here $\varepsilon_{i}= \pm 1$ is determined as follows: If $\phi_{t_{i}+\delta}$, for $\delta>0$ sufficiently small, is a positive resolution of $\phi_{t_{i}}$ then $\varepsilon_{i}=1$. Otherwise $\varepsilon_{i}=-1$.

To prove that $F$ is well defined we have to show that modulo "the integration constant" $F(C L)$, the definition of $F\left(L^{\prime}\right)$ is independent of the choice of the homotopy. For this we consider a closed homotopy $\Psi$ from $C L$ to itself. After a small perturbation, we can assume that there are only finitely many points $x_{1}, x_{2}, \ldots, x_{n} \in S^{1}$, ordered cyclicly according to the orientation of $S^{1}$, so that $\psi_{x_{i}} \in \mathscr{L}^{1}$ and $\psi_{x}$ is equivalent to $\psi_{y}$ for all $x_{i}<x, y<x_{i+1}$. To prove that $F$ is well defined we need to show that

$$
X_{\Psi}:=\sum_{i=1}^{n} \varepsilon_{i} f\left(\psi_{t_{i}}\right)=0
$$

where $\varepsilon_{i}= \pm 1$ is determined by the same rule as above. 
Independence of link component orderings: To prove (6) we will turn our attention to ordered links: First we note that the invariant $f$ pulls back to an invariant on $\widetilde{\mathscr{L}}^{(1)}$ via the forgetful map $r$. After iterating $\Phi$ several times if necessary we can assume that it lifts to a loop in $\mathcal{M}^{L}(P, M)$ based at $L$ (compare p. 3874 of [13]). Given a self-homotopy $\Phi$ of $C L$ and the associated quantity $X_{\Phi}$, lift $\Phi$ to a closed homotopy $\Psi$ in $\mathcal{M}^{L}(P, M)$ and let $X_{\Psi}$ denote the lift of $X_{\Phi}$. Note that $X_{\Psi}=a X_{\Phi}$ for some integer $a \in \mathbb{Z}$. Since $\mathbb{A}$ is torsion free we have $X_{\Phi}=0$ exactly when $X_{\Psi}=0$. Thus, it is enough to check (6) for homotopies that preserve the ordering of components.

Restriction to framing preserving homotopies: Next we observe that it is enough to check (6) for homotopies that are framing preserving in the sense of Definition 2.7: To see that we recall that given a framed link $L^{\prime} \in \mathcal{M}^{C L}(M)$ we need to check that (5) does not depend on the homotopy from $L^{\prime}$ to the framed link $C L$ used to define it. Thus the closed homotopies $\Phi$ that we need (6) to hold for, are those obtained by composing two homotopies from $L^{\prime}$ to $C L$. Each component of $C L$ is equipped with a vector field and going around $\Phi$ does not change the homotopy class of this vector field (that is the equivalence class of $C L$ as a framed link). We can think that the framing of $C L$ transports to a "new" framing around $\Phi$. The two framings might differ by twists on the components of $C L$ but the total singed number of the twists must be zero. The total sum of such twists is captured exactly by the quantity $\Delta \mathbf{f}_{\Phi}$ (compare, Theorem 6 of [13]). The framing of $C L$ lifts to one on $L$ and going around the self-homotopy of $L$ that lifts $\Phi$ also preserves the homotopy class of the framing vector field.

The proof of (6), which occupies the remaining of Section 2 and Section 3, will be divided into several steps. In this section we will give the proof of (6) for closed homotopies in atoroidal 3-manifolds and in the next section we deal with essential tori.

To continue, suppose that $P$ has $m$ components $P=\bigsqcup_{i=1}^{m} P_{i}$, where each $P_{i}$ is an oriented circle. Let $L: P \rightarrow M$ be a link. Pick a base point $p_{i} \in P_{i}$ and let $a_{i}$ denote the homotopy class of $L\left(P_{i}\right)$ in $\pi_{1}\left(M, L\left(p_{i}\right)\right)$. We denote by $Z\left(a_{i}\right)$ the centralizer of $a_{i}$ in $\pi_{1}\left(M, L\left(p_{i}\right)\right)$. We begin with the following lemma (see, for example, the proof of Proposition 4.3 of [21]).

Lemma 2.8. Suppose that $M$ is an orientable 3-manifold with $\pi_{2}(M)=0$ and let the notation be as above. Then

$$
\pi_{1}\left(\mathcal{M}^{L}(P, M), L\right) \cong \bigoplus_{i=1}^{m} Z\left(a_{i}\right) .
$$

2.5. Integrating around inessential tori. Here we show how to derive (6) in the case where the closed homotopy $\Phi$ represents a collection of inessential tori in $M$. Since $\partial M=\emptyset$ this means that the induced map $\left(\Phi_{i}\right)_{*}: \pi_{1}\left(P_{i} \times S^{1}\right) \rightarrow \pi_{1}(M)$ has non-trivial kernel. Here $\Phi_{i}:=\Phi \mid P_{i} \times S^{1}$, for $i=1, \ldots, m$. 
Lemma 2.9. Let $\Phi$ be a loop in $\mathcal{M}^{L}(P, M)$ representing a framing preserving selfhomotopy of L. Suppose that $\Phi$ can be extended to a map $\widehat{\Phi}: P \times D^{2} \rightarrow M$ where $D^{2}$ is a 2-disc with $\partial D^{2}=\{*\} \times S^{1}$. Then $X_{\Phi}=0$.

Proof. We perturb $\widehat{\Phi}$, relatively $\partial D^{2}$, so that it is in general position in the sense of Proposition 1.1 of [14]. Then the set

$$
S_{\widehat{\Phi}}:=\left\{x \in D^{2} \mid \widehat{\phi}_{x}:=\widehat{\Phi}(P \times\{x\}) \text { is not an embedding }\right\}
$$

is a graph in $D^{2}$ with properties (1)-(5) given in Proposition 1.1 of [14]. The vertices of $S_{\widehat{\Phi}}$ in the interior of $D^{2}$ are of valence one or four (see Figure 4).

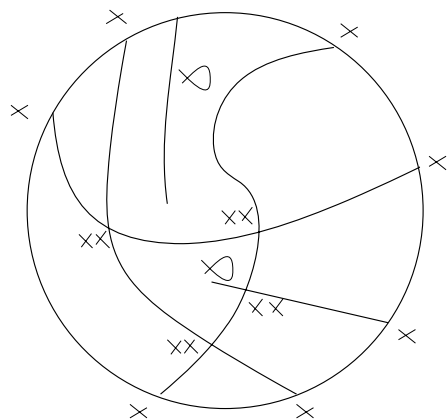

Figure 4 . The set of singularities $S_{\widehat{\Phi}}$ with the types of double points they represent.

The invariant $f$ assigns an element of $\mathbb{A}$ to every edge of $S_{\widehat{\Phi}}$. We observe that condition (3) in the statement of Theorem 2.6 implies that $X_{\Phi}$ is independent on the order in which the crossing changes around $\Phi:=\widehat{\Phi} \mid P \times \partial D^{2}$ occur. Thus, without loss of generality, we may assume that the valence one vertices of $S_{\widehat{\Phi}}$ in the interior of $D^{2}$ correspond to inadmissible crossing changes on $\partial D^{2}$. With the notation as above, we will assume that the framed singular link $\phi_{x_{i}} \in \mathscr{L}^{1}$ is inadmissible for $i=1, \ldots, s$ and admissible for $i=s, \ldots, n$. In particular, there are $s$ edges of $S_{\widehat{\Phi}}$ emanating from $x_{1}, \ldots, x_{s}$ respectively and ending at an interior vertex of valence one, and these are the only valence one vertices of $S_{\widehat{\Phi}}$.

For every interior vertex of $S_{\widehat{\Phi}}$ we draw a small circle $C$ around it so that the number of points in $C \cap S_{\widehat{\Phi}}$ is equal to the valence of the vertex. See Figure 5. Let $C_{1}, \ldots, C_{s}$ denote the circles surrounding the valence one vertices of $S_{\widehat{\Phi}}$ and let $\Gamma$ denote the disjoint union of the circles surrounding the vertices of valence four. For a vertex of valence four the four points in $C \cap S_{\widehat{\Phi}}$ correspond exactly to these appearing in equation (3). Thus by (3) we have

$$
\sum_{x \in \Gamma \cap S_{\widehat{\Phi}}} \varepsilon_{x} f\left(\widehat{\phi}_{x}\right)=0,
$$




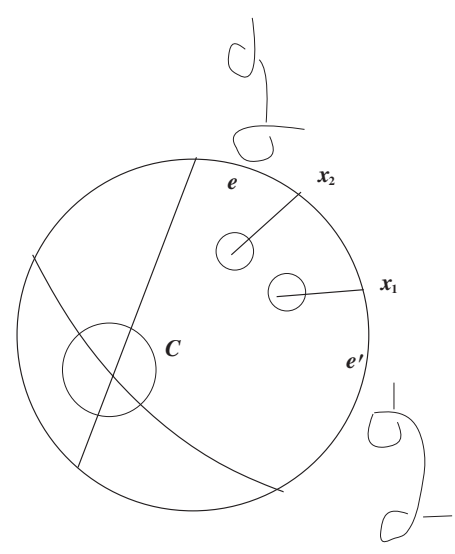

Figure 5. The singular links $\phi_{x_{1}}, \phi_{x_{2}}$ form a pair of type $L_{\times r}, L_{r \times}\left(\right.$ or $\left.L_{\times l}, L_{l \times}\right)$ shown in Figure 3. The framed links corresponding to the components $e, e^{\prime}$ of $\partial D^{2} \backslash\left\{x_{1}, x_{2}, \ldots\right\}$ are isotopic.

where $\widehat{\phi}_{x}:=\widehat{\Phi}(P \times\{x\})$. Now observe that

$$
\sum_{i=s+1}^{n} \varepsilon_{i} f\left(\phi_{x_{i}}\right)=\sum_{x \in \Gamma \cap S_{\widehat{\Phi}}} \varepsilon_{x} f\left(\widehat{\phi}_{x}\right)=0 .
$$

The last equation and (7) imply that

$$
X_{\Phi}=\sum_{i=1}^{s} \varepsilon_{i} f\left(\phi_{x_{i}}\right)
$$

Since $\Phi$ is framing preserving we have $\Delta \mathbf{f}_{\Phi}=\mathbf{0}$. By Definitions 2.3 and 2.7 and the fact that $\mathbf{f}$ remains unchanged under admissible crossing changes we have $\Delta \mathbf{f}_{C}=\mathbf{0}$, for every loop $C \in \Gamma$. This in turn implies that

$$
\Delta \mathbf{f}_{\Gamma}:=\sum_{C \in \Gamma} \Delta \mathbf{f}_{C}=\mathbf{0}
$$

Since we have

$$
\Delta \mathbf{f}_{\Phi}=\sum_{i=1}^{s} \varepsilon_{i} \mathbf{f}\left(\phi_{x_{i}}\right)+\Delta \mathbf{f}_{\Gamma}=\mathbf{0},
$$

we conclude that $\sum_{i=1}^{s} \varepsilon_{i} \mathbf{f}\left(\phi_{x_{i}}\right)=\mathbf{0}$. This in turn implies that the inadmissible singular links $\phi_{x_{i}}$ can be partitioned into pairs of the forms shown in Figure 3. Relation (2) in Remark 2.5 shows that the right-hand side of (8) is identically zero. Thus $X_{\Phi}=0$, as desired.

Remark 2.10. Let $\bar{X}_{\Phi}$ denote the contribution of the admissible singular links around $\Phi$ to $X_{\Phi}$. The proof of Lemma 2.9 shows that regardless of whether $\Phi$ is framing preserving, relation (3) implies that $\bar{X}_{\Phi}=0$. 
Remark 2.11. Proposition 1.1 of [14], referenced in the proof of Lemma 2.9, is stated in there for the PL-category. However, as explained by Kaiser in Section 3 of [12], the statement is true in the smooth category which is actually what we need here. We should also remark that, as explained by Lin in [21], the conclusion holds if the disc $D^{2}$ is replaced by any planar surface $F$. Furthermore, if $\Phi \mid \partial F$ is already in general position then the modifications that put $\Phi$ into general position on $F$ can be performed relatively $\partial F$.

A slight variation of the proof of Lemma 2.9 shows the following.

Lemma 2.12. Let $\Phi$ be a loop in $\mathcal{M}^{L}(P, M)$ representing a framing preserving selfhomotopy of a framed link $L$. Let $P^{\prime}:=P \backslash P_{1}$. Suppose that $\Phi \mid P^{\prime}$ can be extended to a map $\widehat{\Phi}: P^{\prime} \times D^{2} \rightarrow M$ where $D^{2}$ is a 2-disc with $\partial D^{2}=\{*\} \times S^{1}$. Suppose moreover that $\Phi \mid\left(P_{1} \times S^{1}\right)$ is an embedding. Then $X_{\Phi}=0$.

The proof of the next lemma is given in the proof of Lemma 3.3.4 of [16].

Lemma 2.13. Let $M$ be a $\mathbb{Q}$-homology 3-sphere with $\pi_{2}(M)=0$. Suppose that $\pi_{1}(M)$ is infinite and that $L$ has no homotopically trivial components. Let $\Phi \subset$ $\mathcal{M}^{L}(P, M)$ be a framing preserving closed homotopy such that the restriction $\Phi \mid P_{i} \times$ $S^{1} \rightarrow M$ is inessential, for all $i=1, \ldots, m$. There exists a 2 -disc $D^{2}$ and a map $\widetilde{\Phi}: P \times D^{2} \rightarrow M$ such that

$$
X_{\partial \widetilde{\Phi}}=a X_{\Phi}
$$

for some $a \in \mathbb{Z}$. Here $\partial \widetilde{\Phi}=\widetilde{\Phi} \mid P \times \partial D^{2}$.

2.6. Theorem 2.6 for atoroidal manifolds. Before we can proceed with the proof of the theorem we need two additional lemmas.

Lemma 2.14. Consider $\Phi, \Phi^{\prime}: S^{1} \rightarrow \mathcal{M}^{L}(P, M)$ two self-homotopies of L. Let $\bar{X}_{\Phi}$ and $\bar{X}_{\Phi^{\prime}}$ denote the contribution to $X_{\Phi}$ and $X_{\Phi^{\prime}}$ coming from admissible singular links around $\Phi$ and $\Phi^{\prime}$, respectively. Suppose that $\Phi, \Phi^{\prime}$ are freely homotopic as loops in $\mathcal{M}^{L}(P, M)$. Then we have $\bar{X}_{\Phi^{\prime}}=\bar{X}_{\Phi}$. Furthermore, there is a group homomorphism $\psi: \pi_{1}\left(\mathcal{M}^{L}(P, M), L\right) \rightarrow \mathbb{A}$ defined by $\psi([\Phi]):=\bar{X}_{\Phi}$.

Proof. By a slight variation of the argument in the proof of Lemma 3.3.2 of [16] we have the following: There exists a map $\widehat{\Psi}: D^{2} \rightarrow \mathcal{M}^{L}(P, M)$ such that if we set $\Psi:=\widehat{\Psi} \mid \partial D^{2}$, then $\Psi: S^{1} \rightarrow \mathcal{M}^{L}(P, M)$ is a self-homotopy of $L$ with

$$
X_{\Psi}=X_{\Phi}-X_{\Phi^{\prime}}
$$

Lemma 2.9 and Remark 2.10 imply $\bar{X}_{\Psi}=0$; thus $\bar{X}_{\Phi}=\bar{X}_{\Phi^{\prime}}$.

For the remaining of the claim define $\psi: \pi_{1}\left(\mathcal{M}^{L}(P, M), L\right) \rightarrow \mathbb{A}$ as follows: Given $\alpha \in \pi_{1}\left(\mathcal{M}^{L}(P, M), L\right)$, let $\Phi$ is be a self-homotopy of $L$ representing $\alpha$. Define $\psi(\alpha)=\bar{X}_{\Phi}$. By our earlier arguments $\psi(\alpha)$ is independent on the representative $\Phi$. The fact that $\psi$ is a group homomorphism follows easily. 
The next result is Lemma 3.2.5 in [16]. We point out that the proof of this lemma uses the hypothesis that the group $\mathbb{A}$ in which the invariants take values is torsion free.

Lemma 2.15. Suppose that $M$ is a $\mathbb{Q}$-homology 3-sphere with $\pi_{2}(M)=0$. Let $L: P \rightarrow M$ be a framed link and let $\Phi: P \times S^{1} \rightarrow M$ be a framing preserving self-homotopy of $L$. Assume that, for some $i=1, \ldots, m$, we have $a_{i}=1$. Set $P^{\prime}:=P \backslash P_{i}$ and $\Phi^{\prime}:=\Phi \mid P^{\prime}$. If $X_{\Phi^{\prime}}=0$ then $X_{\Phi}=0$.

We are now ready to give the proof of Theorem 2.6 in the case where $M$ is an atoroidal $\mathbb{Q}$-homology 3-sphere.

Theorem 2.16. Suppose that $M$ is an atoroidal $\mathbb{Q}$-homology 3-sphere with $\pi_{2}(M)=0$. Then the conclusion of Theorem 2.6 is true for $M$.

Proof. Let $f: \mathscr{L}^{1} \rightarrow \mathbb{A}$ be a framed singular link invariant satisfying (3) of the statement of Theorem 2.6 and let $\Phi: P \times S^{1} \rightarrow M$ be a framing preserving selfhomotopy of a framed link $L: P \rightarrow M$. We have to show that

$$
X_{\Phi}=0,
$$

where $X_{\Phi}$ is the signed sum of values of $f$ around $\Phi$ defined in (6).

First suppose that $\pi_{1}(M)$ is finite. Then, by Lemma $2.8, \pi_{1}\left(\mathcal{M}^{L}(P, M), L\right)$ is finite. Since $\mathbb{A}$ is torsion free the homomorphism $\psi$ of Lemma 2.14 must be the trivial one. Thus, in particular, $X_{\Phi}=0$.

Now suppose that $\pi_{1}(M)$ is infinite. If the link $L$ to begin with contains no homotopically trivial components, then since $M$ is atoroidal, Lemma 2.13 applies to conclude that $X_{\partial \widetilde{\Phi}}=a X_{\Phi}$, for a map $\widetilde{\Phi}: P \times D^{2} \rightarrow M$. By Lemma 2.9, $X_{\partial \widetilde{\Phi}}=a X_{\Phi}=0$ and thus, since $\mathbb{A}$ is torsion free, $X_{\Phi}=0$.

Next suppose that all the components of $L$ are homotopically trivial; that is $a_{i}=1$, for $i=1, \ldots, m$. Then, by Lemma 2.8 ,

$$
\pi_{1}\left(\mathcal{M}^{L}(P, M), L\right) \cong \bigoplus_{i}^{m} \pi_{1}\left(M, L\left(p_{i}\right)\right) .
$$

Since $H_{1}(M)$ is finite the above equality implies that the abelianization of the group $\pi_{1}\left(\mathcal{M}^{L}(P, M), L\right)$ is a finite group. By Lemma 2.14 we have a homomorphism $\psi: \pi_{1}\left(\mathcal{M}^{L}(P, M), L\right) \rightarrow \mathbb{A}$ with $\psi([\Phi])=X_{\Phi}$. Since $\mathbb{A}$ is abelian $\psi$ factors through the abelianization of $\pi_{1}\left(\mathcal{M}^{L}(P, M), L\right)$; a finite group. But since $\mathbb{A}$ is torsion free $\psi$ is the trivial homomorphism. Thus $X_{\Phi}=0$.

To handle the general case let $h(L)$ denote the number of components of $L$ that are homotopically trivial. The proof is by induction on $h(L)$. In the light of our discussion above, the conclusion is true if $h(L)=0$ or $h(L)=m$. Thus we may assume that $h(L) \neq 0, m$. Let $L_{i} \subset L$ be a component that is homotopically trivial 
and let $L^{\prime}:=L \backslash L_{i}$. Also let $\Phi$ be a self-homotopy of $L$ and let $\Phi^{\prime}$ denote the restriction of $\Phi$ on $P^{\prime}$, where $P^{\prime}:=P \backslash P_{i}$. Since $h\left(L^{\prime}\right)<h(L)$, by induction, $X_{\Phi^{\prime}}=0$. Then, by Lemma 2.15, $X_{\Phi}=0$.

\section{Integration of invariants in toroidal 3-manifolds}

To study the question of integrability of singular link invariants in toroidal 3-manifolds we need several results from the theory of the characteristic submanifold of JacoShalen [10] and Johannson [11]. The statements of the results from these theories, in the form needed in our setting, are summarized in Section 2 of [14] and in Section 2 of [16]. It will be convenient for us to recall the statements we need below from therein, instead from the original references. In particular we will need the Enclosing Theorem and the Torus Theorem both stated on p. 679 of [14]. The later, in the form needed for our purposes, follows from work of Scott, Casson-Jungreis and Gabai.

Theorem 3.1. Let $M$ be a $\mathbb{Z}$-homology 3-sphere with $\pi_{2}(M)=0$ and let $\mathbb{A}$ be a torsion free abelian group. Suppose that a map $f: \mathscr{L}^{(1)} \rightarrow \mathbb{A}$ satisfies (3) of Theorem 2.6. Then there exists a framed link invariant $F$ such that $f$ is derived from $F$ via equation (1).

Remark 3.2. The restriction to $\mathbb{Z}$-homology 3 -spheres in Theorem 3.1 is necessary. As explained in Remark 3.13 of [14] and the discussion at the end of Section 3 in [16], in general, local conditions are not sufficient for the integration of singular link invariants. When the characteristic submanifold contains Seifert fibered components over non-orientable surfaces one needs to impose extra non-local conditions. Specific constructions demonstrating these phenomena are given by Kirk and Livingston in [19]. The necessity of working with irreducible 3-manifolds is demonstrated by [19] as well as the work of Eisermann [7].

The proof of Theorem 2.6 will be completed once we have proved Theorem 3.1. For the proof of Theorem 3.1 we will need the following:

Lemma 3.3. Let $M$ be a $\mathbb{Z}$-homology 3-sphere with $\pi_{2}(M)=0$. Suppose that $\Phi: T=S^{1} \times S^{1} \rightarrow M$ is an essential map. Then there exists a map $\Psi: T \rightarrow M$ homotopic to $\Phi$ and such that one of the following holds:

(1) $\Psi(T)$ lies on an essential embedded torus in $M$.

(2) There exists an oriented surface $F$ with $\partial F \neq \emptyset$, and a trivial fiber bundle $Y=S^{1} \times F$ with the following property: $\Psi$ extends to a map $\hat{\Psi}: Y \rightarrow M$ so that the image $\hat{\Psi}(\partial Y \backslash T)$ is contained on a collection of embedded tori in $M$.

Proof. By the Torus Theorem and the discussion at the end of Section 2 of [16], either $M$ is Haken or it is a Seifert fibered 3-manifold that fibers over $S^{2}$ with three or less exceptional fibers. 
First suppose that $M$ is Haken. Then by the Enclosing Theorem there is a Seifert fibered submanifold $S \subset M$ and a homotopy $\Phi_{t}^{\prime}: T \rightarrow M$ such that $\Phi_{0}^{\prime}=\Phi$ and $\Phi_{1}^{\prime}(T) \subset S$. If $\Phi_{1}^{\prime}(T)$ can be further homotoped in $S$ so that it lies on a component of $\partial S$ then we have conclusion (1). Otherwise, by the classification of essential tori in Haken Seifert fibered spaces (Proposition 2.11 of [14]) we can homotope $\Phi_{1}^{\prime}$ in $S$ to a map $\Psi: T \rightarrow S$ which is vertical with respect to the fibration.

Next suppose that $M$ is a Seifert fibered space. By Proposition 2.2.5 of [16], $\Phi$ is homotopic to a map $\Psi: T \rightarrow M$ which is vertical with respect to the fibration of $M$.

Thus, in both cases, either (1) holds or we have a Seifert fibered manifold $S \subseteq$ $M$, with orbit space $B$ and fiber projection $p$, such that $\Phi$ is homotopic to a map $\Psi: T \rightarrow M$ that is vertical with respect to the fibration of $S$. This means that $\Psi$ is a composition $\Phi_{1} \circ q$, where $q$ is a covering map from the torus $T$ to itself and $\Phi_{1}: T \rightarrow S$ is an immersion without triple points. Then there exists a decomposition $T=S^{1} \times S^{1}$ such that

a) $\Phi_{1}\left(S^{1} \times\{*\}\right)$ maps onto a regular fiber $h$ of $S$;

b) we have $p\left(\Phi_{1}\left(\{*\} \times S^{1}\right)\right)=p\left(\Phi_{1}(T)\right)$ on the orbit surface $B$ of $S$.

Let $H$ (resp. $Q$ ) denote the curve $S^{1} \times\{*\}$ (resp. $\{*\} \times S^{1}$ ) on $T$. Now $\alpha:=p\left(\Phi_{1}(T)\right)$ is an immersed closed curve on $B$ with singularities finitely many transverse double points. A neighborhood $N:=N(\alpha) \subset B$ of $\alpha$ on $B$ is an oriented planar surface. Choose $N$ small enough so that $Y:=p^{-1}(N)$ contains no exceptional fibers of $S$. Now $p: Y \rightarrow N$ is an $S^{1}$-bundle and since $H^{2}(N)=0$ this bundle is trivial. Choose a trivialization $Y \cong S^{1} \times N$ so that $N$ is embedded as a cross-section. Pick a base point $b \in N$ and arcs from $b$ to the components of $\partial N$ whose homotopy classes freely generate $\pi_{1}(N)$; we pick one arc for each such component. Assume that these arcs intersect $\alpha$ only at its double points; let $x_{1}, \ldots, x_{s}$ denote the resulting generators of $\pi_{1}(N, b)$. Write $\alpha$ as a word in these generators, say

$$
[\alpha]=x_{i_{1}}^{k_{1}} x_{i_{2}}^{k_{2}} \ldots x_{i_{r}}^{k_{r}}
$$

We can extend the restriction $\Psi \mid\{*\} \times S^{1}$ to a map $\hat{\Psi}:(F, \partial F) \rightarrow(N, \partial N)$, where $F$ is a planar surface, such that

(i) the induced map $\widehat{\Psi}_{*}: \pi_{1}(F) \rightarrow \pi_{1}(N)$ is onto,

(ii) $\pi_{1}(F)$ is freely generated by elements $a_{1}^{1}, \ldots, a_{k_{1}}^{1}, a_{1}^{2}, \ldots, a_{k_{2}}^{2}, a_{1}^{r}, \ldots, a_{k_{r}}^{r}$,

(iii) $\widehat{\Psi}_{*}([Q])=x_{i_{1}}^{k_{1}} x_{i_{2}}^{k_{2}} \ldots x_{i_{r}}^{k_{r}}$ (see proof of Lemma 3.11 of [14]).

We pull back the fiber bundle structure by $\widehat{\Psi}$ to obtain a fiber bundle $\hat{\Psi}^{*}(Y) \rightarrow F$ over $F$. The pull-back of the cross-section $\alpha$ is a cross-section of $\widehat{\Psi}^{*}(Y)$. Extending this cross-section over $F$, we obtain the conclusion.

We now recall that the proof of Theorem 3.1 is reduced to showing (6) for every framing preserving self-homotopy of $L$. Using Lemmas 2.8, 2.14, and 2.15 we will see that the general case is essentially reduced to the case of knots. Before we continue with the proof Theorem 3.1 some remarks are in order. 
Remark 3.4. Let $\Phi: P \times S^{1} \rightarrow M$ denote a framing preserving self-homotopy of a framed link $L$ and let $\Phi^{\prime}$ be obtained by a free homotopy of $\Phi$ in $M$. Consider the homotopy from $\Phi$ to $\Phi^{\prime}$ as a map $\mathscr{H}: P \times S^{1} \times[0,1] \rightarrow M$. We can smoothly approximate $\mathscr{H}$ by a homotopy in general position as in the proof of Lemma 2.9 (see Remark 2.11). Then we can view $\mathscr{H}$ as a family of smooth framed immersions $S^{1} \rightarrow M$ parametrized by an annulus. We note that the closed homotopy $\Phi^{\prime}$ is not necessarily framing preserving.

Remark 3.5. Suppose that we have a map $\Phi: Y:=S^{1} \times F \rightarrow M$, such that $F$ is a planar surface so that there is a component $\alpha \subset \partial F$ such that the restriction $\Phi \mid S^{1} \times \alpha$ is a loop in $M^{L}(P, M)$. We can view $\Phi$ as a family of framed immersions in $M$, parametrized by $F$. We can cut $Y:=S^{1} \times F \rightarrow M$ along a collection of properly embedded annuli (the projection of which on $F$ decomposes $F$ into a disc) into a product $S^{1} \times D^{2}$. By considering the pull back of $\Phi$ on $S^{1} \times D^{2}$ we obtain a family of framed immersions in $M$ parametrized by $D^{2}$.

In the next lemma we treat homotopies that involve essential tori. The proof treats separately the case of knots and that of links. In the case of knots ( $m=1$ below) the proof is very similar to that of case 1 of Lemma 3.3.3 in [16]. The starting ingredient in the proof of [16] is Lemma 3.3.2 therein. Here we replace that ingredient with Lemma 3.3 and we outline the argument below.

Lemma 3.6. Let $M$ be a $\mathbb{Z}$-homology 3-sphere with $\pi_{2}(M)=0$ and let $\Phi: P \times$ $S^{1} \rightarrow M$ be a framing preserving self-homotopy of a framed link $L$. Suppose that $\Phi_{i}:=\Phi \mid P_{i} \times S^{1}$ is an essential map, for some $i=1, \ldots, m$. Suppose, moreover, that $\Phi_{i}$ cannot be homotoped so that its image lies on an essential embedded torus in $M$. Then we have $X_{\Phi}=0$.

Proof. Let $m$ be the number of components of $L$. We distinguish two cases according to whether $m=1$ or $m>1$.

We have $m=1$ : Since $\Phi$ is framing preserving, relation (2) implies that the total contribution of the inadmissible singular links along $\Phi$ to $X_{\Phi}$ is zero (proof of Lemma 2.9). Thus, without loss of generality, we can assume that no inadmissible crossing changes occur along $\Phi$. Now let $\Psi: P \times S^{1} \rightarrow M$ be a map that is freely homotopic to $\Phi$ in $M$. By Lemma 2.14, and our earlier assumption on $\Phi$, we have $\bar{X}_{\Psi}=X_{\Phi}$.

Set $T:=P \times S^{1}, l:=P \times\{*\}$ and $m:=\{*\} \times S^{1}$. By assumption $\Phi \mid P \times S^{1} \rightarrow$ $M$ is an essential map and it cannot be homotoped so that its image lies on an essential embedded torus in $M$. By Lemma 3.3 we can homotope $\Phi$ to a map $\Psi: P \times S^{1} \rightarrow M$ so that: There is a trivial fiber bundle $Y=S^{1} \times F$, over a planar surface $F$, such that $\Psi$ extends to a map $\hat{\Psi}: S^{1} \times F \rightarrow M$ and the image $\widehat{\Psi}(\partial Y \backslash T)$ is contained on a collection of embedded tori in $M$. Let $H$ denote a simple closed curve $T$ representing a fiber of $Y$ and let $Q$ denote the component of $\partial F$ (embedded as a cross-section of the bundle) on $T$. In $\pi_{1}(T)$ we have $[l]=a[H]+b[Q]$, for some $a, b \in \mathbb{Z}$. 
First suppose that $a=0$. Then Lemma 3.12 of [14] (or Lemma 3.3.1 of [16]) applies to conclude that $\bar{X}_{\Psi}=0$. By our discussion above, $X_{\Phi}=\bar{X}_{\Psi}=0$ and the conclusion in this case follows.

Suppose now that $a \neq 0$. Let $q: \tilde{Y} \rightarrow Y$ be the covering of $Y$ corresponding to the subgroup $a \mathbb{Z} \times \pi_{1}(F)$ of $\pi_{1}(Y)=\mathbb{Z} \times \pi_{1}(F)$. Lift $l, H$, and $Q$ to curves $\tilde{l}, \widetilde{H}$, $\widetilde{Q}$, respectively, on the torus $\widetilde{T}:=q^{-1}(T)$. Now $\widetilde{Y}$ is a trivial fiber bundle over a surface $\widetilde{F}$ with fiber $\tilde{l}$; we will write $\widetilde{Y}=\tilde{l} \times \widetilde{F}$. Consider the composition $\widetilde{\Psi}:=\widehat{\Psi} \circ q$ and its restriction on $\widetilde{T} \cong \tilde{l} \times \widetilde{Q}$. Since $\widetilde{\Psi}(\tilde{l} \times\{x\})=\Psi(l \times q(\{x\}))$, for all $x \in \widetilde{Q}$, the restriction $\widetilde{\Psi} \mid \tilde{l} \times \widetilde{Q}$ is a self-homotopy of a framed knot; the parameter space is $\widetilde{Q}$. As in Remark 3.5 we will think of $\widetilde{\Psi}$ as a family of framed immersions parametrized by a disc $D^{2}$. Then we can consider $X_{\partial \tilde{\Psi}}$. As in the proof of Lemma 3.14 of [14] we obtain that $X_{\partial \tilde{\Psi}}=c X_{\Psi}$, for some $c \in \mathbb{Z}$. Since, as discussed at the beginning of this proof we have $\bar{X}_{\Psi}=X_{\Phi}$, it follows that $\bar{X}_{\partial \tilde{\Psi}}=c X_{\Phi}$. By Remark 2.10, we have $\bar{X}_{\partial \tilde{\Psi}}=0$. Hence we conclude that we have $c X_{\Phi}=0$ for some $c \in \mathbb{Z}$. Since $\mathbb{A}$ is torsion free this implies that $X_{\Phi}=0$; finishing thereby the proof of the Lemma in the case $m=1$.

We have $m>1$ : By Lemma 2.8, $\pi_{1}\left(\mathcal{M}^{L}(P, M), L\right)$ is isomorphic to a direct product of the groups $\pi_{1}\left(\mathcal{M}^{L}\left(P_{i}, M\right), L_{i}\right)$ for $i=1, \ldots, m$. By Lemma 2.14 it is enough to verify (6) only for homotopies $\Phi$ that are fixed on all but one component of $L$. To that end, let $\Psi$ be a homotopy in general position that only moves one component, say $L_{1}$. Suppose, without loss of generality, that $\Psi \mid P_{1} \times S^{1} \rightarrow M$ is an essential map that cannot be homotoped so that its image lies on an essential embedded torus in $M$. By (3), we may decompose $\Psi$ into two homotopies $\Psi_{1}$ and $\Psi_{2}$ such that during $\Psi_{1}$ we only have self-crossing changes on $L_{1}$, while during $\Psi_{2}$ we only have crossing changes between $L_{1}$ and the rest of the components. The argument of case 1 applies to $\Psi_{1}$ to conclude that $X_{\Psi_{1}}=0$. Since the restriction of $\Psi_{2}$ on $P^{\prime} \times S^{1}$, where $P^{\prime}=P \backslash P_{1}$, is constant; it extends to a map $P^{\prime} \times D^{2} \rightarrow M$. Then by Lemma 2.12 we have $X_{\Psi_{2}}=0$.

3.1. The completion of the proof of Theorem 3.1. Let $\Phi$ be a framing preserving loop in $M^{L}(P, M)$. Suppose that $\Phi \mid P_{i} \times S^{1} \rightarrow M$ represents an essential torus for some $i=1, \ldots, m$. First suppose that some component, say $\Phi_{i}:=\Phi \mid P_{i} \times S^{1} \rightarrow M$, can be homotoped to lie on an embedded essential torus in $M$. Then a theorem of Nielsen ([9], Theorem 13.1) implies that after further homotopy, we may assume that $\Phi_{i}$ is a covering map of an embedded torus. It follows that the contribution of $\Phi_{i}$ to $X_{\Phi}$ is zero. Thus, for our purposes, we can assume that if $\Phi_{i}$ induces an injection on $\pi_{1}$ then it cannot be homotoped to lie on an embedded torus. Then by Lemma 3.6 we obtain $X_{\Phi}=0$.

As in the proof of Lemma 3.6 we may assume that $\Phi$ fixes all but one component of $L$, say $L_{1}$. If $\Phi: P_{1} \times S^{1} \rightarrow M$ is inessential the argument in the proof of Theorem 2.16 applies to conclude that $X_{\Phi}=0$. Assume that $\Phi: P_{1} \times S^{1} \rightarrow M$ is essential. Then $X_{\Phi}=0$ by Lemma 3.6. 


\section{Kauffman power series}

4.1. Links in oriented $\mathbb{Q}$-homology 3-spheres. For framed links in $S^{3}$ the Kauffman polynomial is equivalent to a sequence of 1-variable Laurent polynomials $\left\{R_{n}=R_{n}(t)\right\}_{n \in \mathbb{Z}}$ determined by relations

$$
\begin{aligned}
R_{n}(U) & =1, \\
R_{n}\left(L_{r}\right) & =t^{-(n+1)} R_{n}(L), \\
R_{n}\left(L_{l}\right) & =t^{(n+1)} R_{n}(L), \\
R_{n}\left(L_{+}\right)-R_{n}\left(L_{-}\right) & =\left(t-t^{-1}\right)\left[R_{n}\left(L_{o}\right)-R_{n}\left(L_{\infty}\right)\right],
\end{aligned}
$$

where $L_{+}, L_{-}, L_{o}, L_{\infty}$ are as in Figure 1 and $L_{r}, L_{l}$ are as in Figure 2. Notice that the initial value $R_{n}(U)=1$ is just a normalization. Any choice of the initial value together with the rest of the relations will determine a unique $R_{n}$. Set

$$
u_{n}(t):=\frac{t^{n+1}-t^{-(n+1)}}{t-t^{-1}}+1 .
$$

By the relations above one obtains $R_{n}(L \sqcup U)=u_{n}(t) R_{n}(L)$, where the link $L \sqcup U$ is obtained from $L$ by adding an unknotted and unlinked component $U$. The coefficients of the power series $R_{n}(x)$ obtained from $R_{n}(t)$ by substituting $t=e^{x}$ are invariants of finite type [1], [2]. In the theorem below we reverse this procedure and guided by the axioms above we will construct power series invariants generalizing the $R_{n}(x)$ 's: Suppose that $M$ is a $\mathbb{Q}$-homology sphere with $\pi_{2}(M)=0$ and such that if $H_{1}(M) \neq 0$ then $M$ is atoroidal. For every $n \in \mathbb{Z}$ we will construct a sequence of framed link invariants $\left\{v_{n}^{m} \mid m \in \mathbb{N}\right\}$ such that the formal power series

$$
R_{\{M, n\}}=\sum_{m=0}^{\infty} v_{n}^{m} x^{m}
$$

satisfy the axioms above under the change of variable $t=e^{x}$ : We will construct our invariants inductively (induction on $m$ ) by using Theorem 2.6. Each $v_{n}^{m}$ is going to be obtained by integrating a suitable singular link invariant determined by the $v_{n}^{j}$, s with $j<m$. Although the resulting invariants will be invariants of unoriented framed links, for their construction we need to work with oriented links. The reason is that Theorem 2.6 applies to oriented framed links. Recall that $\mathscr{L}$ (resp. $\overline{\mathscr{L}}$ ) denotes the set of isotopy classes of framed oriented (resp. unoriented) links in $M$. Also recall the set of oriented initial links $\mathscr{C} \mathscr{L}:=\mathfrak{v}^{-1}\left(\mathcal{C L}^{*} \cup\{U\}\right)$, defined in the beginning of subsection $\$ 2.3$. By Theorem 2.6 and its proof the invariant $v_{n}^{m}$ is unique once the values on the set $\mathcal{E L}$ are specified.

Theorem 4.1. Assume that $M$ is a $\mathbb{Q}$-homology 3-sphere with $\pi_{2}(M)=0$ and such that if $H_{1}(M, \mathbb{Z}) \neq 0$ then $M$ is atoroidal. Fix $n \in \mathbb{Z}$. Given maps $\mathcal{V}_{n}^{m}: \mathscr{C L}^{*} \cup$ $\{U\} \rightarrow \mathbb{C}, m \in \mathbb{N}$, there exists a unique sequence of complex valued link invariants $\left\{v_{n}^{m} \mid m \in \mathbb{N}\right\}$ with the following properties: 
(1) $v_{n}^{m}(C L)=\mathcal{V}_{n}^{m}(\mathfrak{D}(C L))$ for all $C L \in \mathcal{C L}$ and $m \in \mathbb{N}$.

(2) $v_{n}^{m}(L)=v_{n}^{m}(\mathfrak{D}(L))$ for all $L \in \mathscr{L}$ and $m \in \mathbb{N}$. Thus the values of the invariants are independent of the link orientation.

(3) If we define a formal power series

$$
R_{n}:=R_{\{M, n\}}(L)=\sum_{m=0}^{\infty} v_{n}^{m}(L) x^{m}
$$

then we have

$$
\begin{aligned}
R_{n}(U) & =1, \\
R_{n}\left(L_{r}\right) & =t^{-(n+1)} R_{n}(L), \\
R_{n}\left(L_{l}\right) & =t^{(n+1)} R_{n}(L), \\
R_{n}\left(L_{+}\right)-R_{n}\left(L_{-}\right) & =\left(t-t^{-1}\right)\left[R_{n}\left(L_{o}\right)-R_{n}\left(L_{\infty}\right)\right],
\end{aligned}
$$

where $t:=e^{x}=1+x+\frac{x^{2}}{2}+\cdots$.

Proof. Define $v_{n}^{m}(C L)=\mathcal{V}_{n}^{m}(\mathfrak{o}(C L))$ for all $C L \in \mathscr{C L}$ and $m \in \mathbb{N}$. Now we can form the power series $R_{n}(C L)$. Guided by (12)-(13) we define

$$
R_{n}\left(C L_{r}\right)=t^{-(n+1)} R_{n}(C L) \text { and } R_{n}\left(C L_{l}\right)=t^{(n+1)} R_{n}(C L) .
$$

Now guided by these we can define the values of $R_{n}$ on all framed links whose underlying unframed isotopy class is $C L$. To explain this suppose that $C L$ has $S$ components. Let $C L(\mathbf{f})$ be a framed link in the same (unframed) isotopy class with $C L$ with framing unordered sequence $\mathbf{f}$ (see Definition 2.2 and preceding discussion). Then define

$$
R_{n}(C L(\mathbf{f}))=t^{(n+1) \tau} R_{n}(C L),
$$

where $\tau:=\tau(C L(\mathbf{f}))$ is the total framing of $C L(\mathbf{f})$. Using (14)-(15), and inducting on $k$, we can check that

$$
R_{n}\left(C L \sqcup U^{k}\right)=\left[u_{n}(t)\right]^{k-1} R_{n}(C L),
$$

where $u_{n}(t)$ is given by (10). Now $R_{n}$ has been defined on all framed links in the unframed isotopy classes of the links in $\mathscr{C L}$.

To continue for every $L(\mathbf{f}) \in \mathscr{L}$ with framing sequence $\mathbf{f}$ we define

$$
v_{n}^{0}(L(\mathbf{f}))=v_{n}^{0}(C L(\mathbf{f}))
$$

where $C L$ is the initial link homotopic to $L$. Inductively, suppose that the invariants $v_{n}^{0}, v_{n}^{1}, \ldots, v_{n}^{m-1}$ have been defined such that if we let

$$
R_{n}^{(m-1)}(L):=\sum_{i=1}^{m-1} v_{n}^{i}(L) x^{i},
$$


then we have

$$
\begin{aligned}
R_{n}^{(m-1)}\left(L_{r}\right) & =t^{-(n+1)} R_{n}^{(m-1)}(L) \bmod x^{m}, \\
R_{n}^{(m-1)}\left(L_{l}\right) & =t^{(n+1)} R_{n}^{(m-1)}(L) \bmod x^{m}, \\
R_{n}^{(m-1)}(L \sqcup U) & =u_{n}(t) R_{n}(L) \bmod x^{m},
\end{aligned}
$$

and

$$
R_{n}^{(m-1)}\left(L_{+}\right)-R_{n}^{(m-1)}\left(L_{-}\right)=\left(t-t^{-1}\right)\left[R_{n}^{(m-1)}\left(L_{o}\right)-R_{n}^{(m-1)}\left(L_{\infty}\right)\right] \bmod x^{m} .
$$

Furthermore, suppose that these invariants do not depend on the orientation of the links. The last equation leads us to define

$$
R_{n}^{(m)}\left(L_{\times}\right):=\left(t-t^{-1}\right)\left[R_{n}^{(m-1)}\left(L_{o}\right)-R_{n}^{(m-1)}\left(L_{\infty}\right)\right] \bmod x^{m} .
$$

We want to define the invariant $v_{m}^{n}$ : Recall that it is already defined on the initial links. Next we examine the right-hand side of (20). It is a polynomial of degree $m$ such that the coefficient of $x^{m}$ comes from

$$
\left(t-t^{-1}\right)\left[R_{n}^{(m-1)}\left(L_{o}\right)-R_{n}^{(m-1)}\left(L_{\infty}\right)\right] .
$$

The expression above has no constant term and thus the coefficient of $x^{m}$ depends on the inductively well-defined invariants $v_{n}^{i}, i=1,2, \ldots, m-1$. Thus the coefficient of $x^{m}$ in (20) is a "new" framed singular link invariant. We are going to prove that it is derived from a framed link invariant by using Theorem 2.6. For that we need to check that condition (3) in Theorem 2.6 is satisfied. It is enough to check it modulo $x^{m+1}$. In what follows the symbol " $\equiv$ " will denote calculation modulo $x^{m+1}$.

Let $L_{x+}$ and $L_{\times-} \in \mathscr{L}^{(1)}$ be two singular framed links as in the left-hand side of (3) in Theorem 2.6. From (20) we have

$$
\begin{aligned}
R_{n}^{(m)}\left(L_{\times+}\right)-R_{n}^{(m)}\left(L_{\times-}\right) \equiv & \left(t-t^{-1}\right)\left[R_{n}^{(m-1)}\left(L_{o+}\right)-R_{n}^{(m-1)}\left(L_{\infty+}\right)\right] \\
& -\left(t-t^{-1}\right)\left[R_{n}^{(m-1)}\left(L_{o-}\right)-R_{n}^{(m-1)}\left(L_{\infty-}\right)\right] \\
\equiv & \left(t-t^{-1}\right)\left[R_{n}^{(m-1)}\left(L_{o+}\right)-R_{n}^{(m-1)}\left(L_{o-}\right)\right] \\
& -\left(t-t^{-1}\right)\left[R_{n}^{(m-1)}\left(L_{\infty+}\right)-R_{n}^{(m-1)}\left(L_{\infty-}\right)\right] \\
\equiv & \left(t-t^{-1}\right)^{2}\left[R_{n}^{(m-1)}\left(L_{o o}\right)-R_{n}^{(m-1)}\left(L_{o \infty}\right)\right] \\
& -\left(t-t^{-1}\right)^{2}\left[R_{n}^{(m-1)}\left(L_{\infty o}\right)-R_{n}^{(m-1)}\left(L_{\infty}\right)\right] \\
\equiv & \left(t-t^{-1}\right)^{2}\left[R_{n}^{(m-1)}\left(L_{o o}\right)+R_{n}^{(m-1)}\left(L_{\infty}\right)\right] \\
& -\left(t-t^{-1}\right)^{2}\left[R_{n}^{(m-1)}\left(L_{\infty o}\right)+R_{n}^{(m-1)}\left(L_{o \infty}\right)\right] .
\end{aligned}
$$

Since the result is symmetric with respect to the two double points we deduce that

$$
R_{n}^{(m)}\left(L_{\times+}\right)-R_{n}^{(m)}\left(L_{\times-}\right) \equiv R_{n}^{(m)}\left(L_{+\times}\right)-R_{n}^{(m)}\left(L_{-\times}\right) .
$$


Thus the framed singular link invariant defined above is induced by a framed link invariant. Recall that we have already defined the values of $v_{n}^{m}$ on all framed links with unframed underlying isotopy classes in $\mathscr{C L}$. Using this values we can define a link invariant $v_{n}^{m}$ for all links in $\mathscr{L}$ such that if we let

$$
R_{n}^{(m)}(L)=\sum_{i=1}^{m} v_{n}^{m}(L) x^{i}
$$

we have

$$
R_{n}^{(m)}\left(L_{+}\right)-R_{n}^{(m)}\left(L_{-}\right)=R_{n}^{(m)}\left(L_{\times}\right)
$$

for every $L_{\times} \in \mathscr{L}^{(1)}$. Now it is a straightforward calculation to check that the inductive hypotheses hold mod $x^{m+1}$. For example let us check (18); the others are similar. Consider a framed link $L_{r}$. Keeping the kink intact in a small 3-ball, make a sequence of crossing changes to transform $L_{l}$ to an initial link say $C L_{l}$. Over all such sequences of crossing changes, and initial links $C L_{l}$, choose one that minimizes the number of the required crossing changes. Suppose, without loss of generality, that the first crossing to be changed in that sequence is a positive crossing. By (20) and (21) we have

$$
R_{n}^{(m)}\left(L_{l+}\right) \equiv R_{n}^{(m)}\left(L_{l-}\right)+\left(t-t^{-1}\right)\left[R_{n}^{(m-1)}\left(L_{l o}\right)-R_{n}^{(m-1)}\left(L_{l \infty}\right)\right] \bmod x^{m+1} .
$$

By (15) and induction on the number of crossing changes needed to go from $L_{l+}$ to $C L_{l}$ we can assume that

$$
R_{n}^{(m)}\left(L_{l-}\right) \equiv t^{(n+1)} R_{n}^{(m)}(L)
$$

By (18) we have

$$
R_{n}^{(m-1)}\left(L_{l o}\right)=t^{(n+1)} R_{n}^{(m-1)}\left(L_{o}\right) \bmod x^{m}
$$

and

$$
R_{n}^{(m-1)}\left(L_{l \infty}\right)=t^{(n+1)} R_{n}^{(m-1)}\left(L_{\infty}\right) \bmod x^{m} .
$$

Combining the last four equations we have

$$
R_{n}^{(m)}\left(L_{l+}\right) \equiv t^{(n+1)} R_{n}^{(m)}\left(L_{+}\right),
$$

as desired. To finish the proof we need to show that $v_{n}^{m}$ is independent of the link orientation. Inductively, we assume that $v_{n}^{0}, v_{n}^{1}, \ldots, v_{n}^{m-1}$ are uniquely determined by their values on $\mathcal{E L}$ and independent of the (singular) link orientation. We have that

$$
v_{n}^{m}(L)=v_{n}^{m}(C L)+\sum_{i=1}^{s} \pm v_{n}^{m}\left(L_{i}\right),
$$

where $L_{1}, \ldots, L_{s} \in \mathscr{L}^{(1)}$ are singular links appearing in a homotopy from $L$ to $C L$, where $C L$ is the representative of $L$ in $\mathscr{C L}$ (compare relation (5)). Recall that we 
defined $v_{n}^{m}(C L)$ to be independent of the orientation for $C L$. The proof of Theorem 2.6 establishes that $v_{n}^{m}(L)$ does not depend on the homotopy from $L$ to $C L$ chosen. By induction $v_{n}^{0}, v_{n}^{1}, \ldots, v_{n}^{m-1}$ do not depend on orientations. It follows that $v_{n}^{m}(L)$ is unique once $v_{n}^{m}(C L)$ is chosen and independent on link orientation.

Theorem 1.6 stated in the Introduction is obtained from Theorem 4.1 if we set $z:=i t-(i t)^{-1}=i e^{x}+i e^{-x}$ and $a:=i e^{y}$, where $y=(n+1) x$. Now we derive Theorem 1.5 stated in the Introduction.

Proof of Theorem 1.5. The elements in the set $\mathcal{C} \mathscr{L}^{*} \cup\{U\}$ are in one-to-one correspondence with a basis of $S(\hat{\pi})$. An element $R \in \mathfrak{F}^{*}(M)$ gives rise to one in $S^{*}(\hat{\pi})$ by restriction on the set $\mathcal{C} \mathscr{L}^{*} \cup\{U\}$. Thus one direction of the theorem follows. For the other direction, we note that an element in $S^{*}(\hat{\pi})$ defines a map $\mathcal{R}_{M}: \mathcal{C L} \mathscr{L}^{*} \cup\{U\} \rightarrow \hat{\Lambda}$. Then by Theorem 1.6 there is a unique map $R_{M}: \overline{\mathscr{L}} \rightarrow \hat{\Lambda}$ with properties (1)-(3). These properties guarantee that $R_{M}$ factors through the Kauffman module $\mathfrak{F}(M)$ to give an element in $\mathfrak{F}^{*}(M)$ (see Definition 1.2).

4.2. Links in $S^{3}$. Links in $S^{3}$ are studied via projections on a sphere $S^{2} \subset S^{3}$. Let $U^{m}$ denote the standard $m$-component unlink and $U^{m}(\mathbf{f})$ denote the one with framing $\mathbf{f}$. Every $m$-component link projection $L \subset S^{2}$ is transformed to a framed unlink by finitely many crossing changes and regular isotopy moves on $S^{2}$ (i.e. isotopy using the Reidemeister moves of type II and III only). For a link projection $L \subset S^{2}$, we define a complexity

$$
s(L):=(u(L), c(L))
$$

as follows: $c(L)$ is the number of crossings of $L$ and $u(L)$ is the number of admissible crossing changes required to transform $L$ into a diagram of the unlink that admits a type I Reidemeister move that reduces its crossing number. We order the complexities lexicographically. Let $R:=R_{S^{3}}: \mathscr{L} \rightarrow \hat{\Lambda}$ be a map constructed as in Theorem 1.6 and recall that $\Lambda:=\mathbb{C}\left[a^{ \pm 1}, z^{ \pm 1}\right]$. Note that the complexity $s(L)$ defined above has the properties that $s\left(L_{r}\right), s\left(L_{1}\right)>s(L)$.

Proposition 4.2. Define $R(U(\mathbf{f}))=a^{-\tau}\left(a+a^{-1}\right) z^{-1}+1$, where $\tau:=\sum_{i=1}^{m} \mathbf{f}_{\mathbf{i}}$. Then $R(L) \in \Lambda$ for every link. In fact, $R(L)$ is the two variable Kauffman polynomial.

Proof. Given a diagram $L$ we can first perform all type I Reidemeister moves that reduce the number of crossings of $L$. If there are no such moves, and $L$ is not the unlink, then there is a crossing change such that three of the terms $s\left(L_{-}\right), s\left(L_{o}\right)$, $s\left(L_{\infty}\right), s\left(L_{+}\right)$are strictly less that the remaining fourth one. Thus the skein relations

$$
\begin{aligned}
R\left(L_{+}\right)-R\left(L_{-}\right) & =z\left[R\left(L_{o}\right)-R\left(L_{\infty}\right)\right], \\
R\left(L_{r}\right) & =a R(L), \\
R\left(L_{l}\right) & =a^{-1} R(L)
\end{aligned}
$$


allow us to write the invariant $R(L)$ of every link $L$ as a finite sum of the invariants of links of strictly less complexity than $s(L)$ and with coefficients in $\Lambda$. The result follows by induction on $s(L)$ and the observation that $R(U(\mathbf{f})) \in \Lambda$. The last claim follows by the uniqueness properties of $R$.

\section{References}

[1] D. Bar-Natan, On the Vassiliev knot invariants. Topology 34 (1995), 423-472. MR 1318886 Zbl 0898.57001

[2] J. S. Birman and X.-S. Lin, Knot polynomials and Vassiliev's invariants. Invent. Math. 111 (1993), 225-270. MR 1198809 Zbl 0812.57011

[3] V. Chernov, Framed knots in 3-manifolds and affine self-linking numbers. J. Knot Theory Ramifications 14 (2005), 791-818. MR 2172898 Zbl 1083.57016

[4] C. Cornwell, A polynomial invariants for links in lens spaces. Preprint 2010. arXiv:1002.1543 [math.GT]

[5] C. Cornwell, Bennequin type inequalities in lens spaces. Preprint 2010. arXiv:1002.1546 [math.GT]

[6] C. Cornwell, Ph.D. thesis, Michigan State University East Lansing, in preparation.

[7] M. Eisermann, A geometric characterization of Vassiliev invariants. Trans. Amer. Math. Soc. 355 (2003), 4825-4846. MR 1997586 Zbl 1033.57005

[8] E. Ferrand, On Legendrian knots and polynomial invariants. Proc. Amer. Math. Soc. 130 (2002), 1169-1176. MR 1873793 Zbl 1005.53023

[9] J. Hempel, 3-manifolds. Ann. of Math. Stud. 86, Princeton University and University of Tokyo Press, Princeton, N.J., Tokyo 1976. MR 0415619 Zbl 0345.57001

[10] W. H. Jaco and P. B. Shalen, Seifert fibered spaces in 3-manifolds. Mem. Amer. Math. Soc. 21 (1979), no. 220. MR 539411 Zbl 0415.57005

[11] K. Johannson, Homotopy equivalences of 3-manifolds with boundaries. Lecture Notes in Math. 761, Springer, Berlin 1979. MR 0551744 Zbl 0412.57007

[12] U. Kaiser, Presentations of homotopy skein modules of oriented 3-manifolds. J. Knot Theory Ramifications 10 (2001), 461-491. MR 1825969 Zbl 0994.57016

[13] U. Kaiser, Quantum deformations of fundamental groups of oriented 3-manifolds. Trans. Amer. Math. Soc. 356 (2004), 3869-3880. MR 2058509 Zbl 1054.57009

[14] E. Kalfagianni, Finite type invariants for knots in 3-manifolds. Topology 37 (1998), 673707. MR 1604915 Zbl 0911.57008

[15] E. Kalfagianni, Power series link invariants and the Thurston norm. Topology Appl. 101 (2000), 107-119. MR 1732063 Zbl 0944.57005

[16] E. Kalfagianni and X.-S. Lin, The HOMFLY polynomial for links in rational homology 3-spheres. Topology 38 (1999), 95-115. MR 1644083 Zbl 0961.57005

[17] L. H. Kauffman, An invariant of regular isotopy. Trans. Amer. Math. Soc. 318 (1990), 417-471. MR 958895 Zbl 0763.57004 
[18] L. H. Kauffman, Knots and physics. 3rd ed., Ser. Knots Everything 1, World Scientific Publishing Co., Singapore 2001. MR 1858113 Zbl 1057.57001

[19] P. Kirk and C. Livingston, Knot invariants in 3-manifolds and essential tori. Pacific J. Math. 197 (2001), 73-96. MR 1810209 Zbl 1045.57004

[20] T. T. Q. Le, J. Murakami, and T. Ohtsuki, On a universal perturbative invariant of 3manifolds. Topology 37 (1998), 539-574. MR 1604883 Zbl 0897.57017

[21] X.-S. Lin, Finite type link invariants of 3-manifolds. Topology 33 (1994), 45-71. MR 1259514 Zbl 0816.57013

[22] J. H. Przytycki, A $q$-analogue of the first homology group of a 3-manifold. In Perspectives on quantization (South Hadley, MA, 1996), Contemp. Math. 214, Amer. Math. Soc., Providence, RI, 1998, 135-144. MR 1601241 Zbl 1004.57020

[23] J. Przytycki, Skein modules. Preprint 2006. arXiv:math/0602264 [math.GT]

[24] N. Reshetikhin and V. G. Turaev, Invariants of 3-manifolds via link polynomials and quantum groups. Invent. Math. 103 (1991), 547-597. MR 1091619 Zbl 0725.57007

[25] D. Rutherford, Thurston-Bennequin number, Kauffman polynomial, and ruling invariants of a Legendrian link: the Fuchs conjecture and beyond. Internat. Math. Res. Notices 2006 (2006), Art. ID 78591. MR 2219227 Zbl 1106.57012

[26] S. Tabachnikov, Estimates for the Bennequin number of Legendrian links from state models for knot polynomials. Math. Res. Lett. 4 (1997), 143-156. MR 1432817 Zbl 0877.57001

[27] V. A. Vassiliev, Cohomology of knot spaces. In Theory of singularities and its applications, Adv. Soviet Math. 1, Amer. Math. Soc., Providence, RI, 1990, 23-69. MR 1089670 Zbl 0727.57008

[28] V. A. Vassiliev, On invariants and homology of spaces of knots in arbitrary manifolds. In Topics in quantum groups and finite-type invariants, Amer. Math. Soc. Transl. Ser. (2) 185, Amer. Math. Soc., Providence, RI, 1998, 155-182. MR 1736171 Zbl 0929.57004

[29] E. Witten, Quantum field theory and the Jones polynomial. Comm. Math. Phys. 121 (1989), 351-399. MR 990772 Zbl 0667.57005

Received February 23, 2010

E. Kalfagianni, Department of Mathematics, Michigan State University, East Lansing, MI 48824, U.S.A.

E-mail: kalfagia@math.msu.edu 